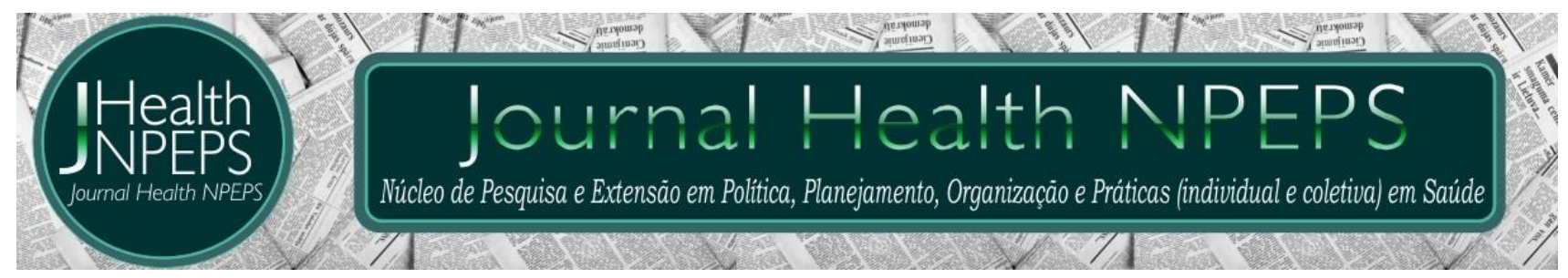

http://dx.doi.org/10.30681/252610104707

ARTIGO ORIGINAL

\title{
Perfil clínico e epidemiológico de vítimas de acidentes por animais peçonhentos em Santarém - PA
}

\section{Epidemiological profile and clinical management of accidents by venomous animals in Santarém - PA}

\section{Perfil clínico y epidemiológico de las víctimas de accidentes por animales venenosos en Santarém - PA}

\section{Luan Duarte Lopes ${ }^{1}$, João David Batista Lisbôa², Flávia Garcez da Silva³}

\section{RESUMO}

Objetivo: identificar o perfil clínico e epidemiológico de vítimas de acidentes por animais peçonhentos no município de Santarém, Pará. Método: estudo quantitativo, descritivo e transversal, utilizando dados do Sistema de Informação de Agravos de Notificação (SINAN), da Divisão de Vigilância em Saúde de Santarém (DIVISA) e informações sobre o manejo clínico realizado no Hospital Municipal de Santarém. Resultados: foram notificados 2658 no SINAN e registrados 2754 casos na DIVISA, ocorrendo predominantemente nos meses de janeiro, setembro e outubro. A maioria dos casos foram indivíduos do sexo masculino, entre 20 a 64 anos. Os tipos de acidentes foram provenientes principalmente de serpentes e escorpiões. A maior parte dos acidentes foi classificada como "moderada", evoluindo para cura. Conclusão: os dados mostraram que existem possíveis falhas na notificação desses acidentes devido à expressividade da categoria "ignorado/branco". Além disso, constatou-se uma inconsistência entre as duas fontes de dados (SINAN e DIVISA).

Descritores: Ecossistema Amazônico; Antivenenos; Animais Venenosos.

\section{ABSTRACT}

Objective: to identify the clinical and epidemiological profile of victims of accidents caused by poisonous animals in the municipality of Santarém, Pará. Method: quantitative, descriptive and cross-sectional study, using data from the Notifiable Diseases Information System (SINAN) from the Santarém Health Surveillance Division

\footnotetext{
${ }^{1}$ Acadêmico de Farmácia. Universidade Federal do Oeste do Pará. Santarém, Pará, Brasil. E-mail: luanfarma2013@gmail.com ORCID ID: https://orcid.org/0000-0001-5604-7295 Autor para correspondência Endereço: Rua Vitória, 407-Caranazal, Santarém - PA - Brasil, CEP 6804-0305.

${ }^{2}$ Farmacêutico. Mestre em Sociedade, Ambiente e Qualidade de Vida. Residente da Universidade Federal do Oeste do Pará Santarém, Pará, Brasil. E-mail: joaodblisboa@gmail.com ORCID ID: https://orcid.org/0000-0003-2885-1360

${ }^{3}$ Farmacêutica. Doutora em Toxicologia. Docente da Universidade Federal do Oeste do Pará. Santarém, Pará, Brasil. Email: fgarcez@yahoo.com.br ORCID ID: https://orcid.org/0000-0002-0513-6017
}

Este artigo está licenciado sob forma de uma licença Creative Commons Atribuição 4.0 Internacional, que permite uso irrestrito, distribuição e reprodução em qualquer meio, desde que a publicação original seja corretamente citada 
(DIVISA), and information on clinical management carried out at the Santarem Municipal Hospital. Results: 2658 were reported in SINAN and 2754 cases were registered in DIVISA, occurring predominantly in the months of January, September and October. Most cases were male, between 20 and 64 years old. The types of accidents came mainly from snakes and scorpions. Most accidents were classified as "moderate", evolving to cure. Conclusion: the data showed that there are possible failures in reporting these accidents due to the expressiveness of the "ignored / white" category. In addition, there was an inconsistency between the two data sources (SINAN and DIVISA).

Descriptors: Amazonian Ecosystem; Antivenins; Animals, Poisonous.

\section{RESUMEN}

Objetivo: Identificar el perfil clínico y epidemiológico de las víctimas de accidentes causados por animales venenosos en el municipio de Santarém, Pará. Método: Estudio cuantitativo, descriptivo y transversal, utilizando datos del Sistema de Información de Enfermedades Notificables (SINAN), de la División de Vigilancia Sanitaria de Santarém (DIVISA) e información de gestión clínica realizada en el Hospital Municipal de Santarém. Resultados: en el SINAN se notificaron 2658 y en la DIVISA se registraron 2754 casos, ocurriendo predominantemente en los meses de enero, septiembre y octubre. La mayoría de los casos fueron de sexo masculino, entre 20 y 64 años. Los tipos de accidentes provinieron principalmente de serpientes y escorpiones. La mayoría de los accidentes se clasificaron como "moderados", evolucionando para curar. Conclusión: los datos mostraron que existen posibles fallas en la notificación de estos accidentes debido a la expresividad de la categoría "ignorados / blancos". Además, hubo una inconsistencia entre las dos fuentes de datos (SINAN y DIVISA).

Descriptores: Ecosistema Amazónico; Antivenenos; Animales Venenosos.

\section{INTRODUÇÃOO}

Os animais peçonhentos são biologicamente capazes de produzir e armazenar substâncias potencialmente tóxicas em glândulas para inoculá-las em suas presas ou predadores, através de aparelhos excretores como dentes modificados, aguilhões e ferrões $^{1,2}$. Estima-se que existam mais de 100 mil espécies peçonhentas espalhadas pelo mundo ${ }^{3}$ com incidência maior em países tropicais e subtropicais. Os latinoamericanos se destacam por possuírem maior diversidade de habitats ${ }^{4}$.
No Brasil, os escorpiões lideram as causas de acidentes por animais peçonhentos, sendo as espécies com mais casos graves: Tityus bahiensis, Tityus serrulatus e Tityus stigmurus ${ }^{5,6}$. Dentre as serpentes estão Bothrops ssp. (jararaca); Crotalus ssp. (cascavel); Lachesis ssp. (surucucu pico-de-jaca) e Micrurus ssp. (coral verdadeira) ${ }^{7}$. Enquanto as aranhas de maior interesse para a saúde pública são do gênero Phoneutria (aranha-armadeira), Latrodectus (viúva-negra) e Loxosceles (aranha-marrom) $^{8}$. 
$\mathrm{Na}$ Amazônia, os principais animais peçonhentos registrados são as serpentes, escorpiões, lagartas, abelhas, aranhas, lacraias, formigas, vespas e arraias $^{9}$. Os acidentes provocados por esses animais possuem grande importância no mundo, devido a sua frequente ocorrência, grande número de casos, gravidade clínica, podendo levar a morbidade e/ou mortalidade 7,9,10.

O manejo clínico das vítimas acidentadas por animais venenosos segue um protocolo preconizado pelo Ministério da Saúde, podendo sofrer alterações de acordo com a região em que acontecem esses acidentes ${ }^{11}$. 0 fluxo desse manejo acontece $\mathrm{da}$ seguinte forma: primeiramente 0 paciente é estabilizado (avaliação dos sinais vitais), verifica-se 0 histórico (local da picada, tempo aproximado decorrido, se fez usa de garrote ou usou algum remédio) e identificação da espécie causadora. 0 tratamento consiste na higienização do local da picada, controle dos sintomas e administração do soro antiveneno, quando necessário ${ }^{12}$.

Através de estudos epidemiológicos, é possível compreender o processo saúde-doença na população, avaliando a distribuição, a frequência e os fatores determinantes relacionados a danos à saúde coletiva ${ }^{13}$. Uma análise dos acidentes causados por animais peçonhentos possibilita a implementação de métodos eficazes de prevenção, controle e identificação dos $\operatorname{casos}^{14}$. 0 tratamento consiste majoritariamente na administração de soroterapia antivenenos, pois é o único cientificamente capaz de neutralizar a ação das substâncias tóxicas injetadas por esses animais, através de anticorpos específicos $^{15,16}$.

Atualmente, o Brasil conta com Sistema de Informação de agravos de Notificação (SINAN), Sistema Nacional de Informações Tóxico-Farmacológicas (SINITOX/FIOCRUZ), Sistema de Informações Hospitalares do Sistema Único de Saúde e o Sistema de Informação sobre Mortalidade (SIM), para as notificações de acidentes por animais peçonhentos ${ }^{17}$.

Segundo dados do SINAN, o Estado do Pará se destaca em notificações de acidentes de animais peçonhentos na região Norte. No ano de 2017, a região notificou aproximadamente 17.340 novos casos de acidentes por animais peçonhentos, sendo 7.520 casos no estado do Pará ${ }^{18}$. Nesse sentido, este artigo tem como objetivo identificar o perfil clínico e epidemiológico de vítimas de acidentes 
por animais peçonhentos no município de Santarém, Pará.

\section{MÉTODO}

Trata-se de um estudo quantitativo, descritivo e transversal pautado em dados de acidentes com animais peçonhentos ocorridos no munícipio de Santarém, Pará, no período de janeiro de 2012 a dezembro de 2017. Utilizou-se duas fontes de dados de domínio público, disponibilizados pelo Sistema de Informação de Agravos de Notificação (SINAN) e pela Secretaria Municipal de Saúde de Santarém, Pará.

O Sistema de Informação de Agravos de Notificação (SINAN) é responsável por coletar, transmitir e disseminar dados de doenças e agravos de notificação compulsória nacional. As variáveis observadas nesse sistema foram: mês e ano; gênero; faixa etária; escolaridade; tipo de agente e tipo de serpente; classificação dos casos quanto à gravidade; tempo decorrido entre o acidente e $o$ atendimento e evolução dos casos.

Os dados epidemiológicos obtidos na Secretaria Municipal de Saúde de Santarém, foi por meio da Divisão de Vigilância em Saúde (DIVISA), que é responsável por receber as fichas de notificações das Unidades de Saúde e transmiti-las para o SINAN. Já as informações a respeito do manejo clínico dos acidentes por animais peçonhentos foram disponibilizadas pelo Hospital Municipal de Santarém (HMS).

As variáveis observadas na DIVISA foram: residência; anatomia do local da picada; frequência de manifestações locais; alteração da coagulação e administração de soroterapia. Para a criação do fluxo do manejo clínico foi analisado o protocolo de conduta utilizado pelo setor de urgência/emergência do HMS das vítimas acidentadas por animais peçonhentos.

Os dados foram coletados entre agosto e dezembro de 2019, transcritos utilizando o programa Microsoft Excel 2010, para a sistematização em planilhas, montagem de gráficos e tabelas. E analisados por estatística descritiva simples. As variáveis quantitativas foram descritas pelo seu valor absoluto e por meio da distribuição de frequências relativas.

As estimativas populacionais empregadas para o cálculo do coeficiente de incidência foram obtidos do Instituto Brasileiro de Geografia e Estatística (IBGE). Em 2012 a estimativa era de 286.343 mil habitantes no município de Santarém, em 2013 de 
288.462 mil habitantes, em 2014 de 290.521 mil habitantes, no ano de 2015 eram 292.520 mil habitantes, em 2016 um total de 294.447 mil habitantes e em 2017 foram estimados 296.302 mil habitantes no município.

Santarém está localizada na região do Baixo Amazonas, constituída por uma ampla área urbana e por diversas comunidades oriundas de planaltos, florestas e rios. As principais atividades econômicas dessa região são a mineração, agricultura e turismo. 0 turismo se destaca devido aos atrativos naturais da região, principalmente praias, como Alter-do-Chão, conhecida como o "Caribe Amazônico".

0 presente estudo seguiu todas as normas de éticas em pesquisa, sendo submetido e aprovado pelo Comitê de Ética em Pesquisa de acordo com CAAE: 99005918.8.0000.5168 e número do parecer 3.078 .530 no dia 28 de dezembro de 2018.

Figura 1 - Localização geográfica do estudo epidemiológico Santarém, Pará, Brasil.

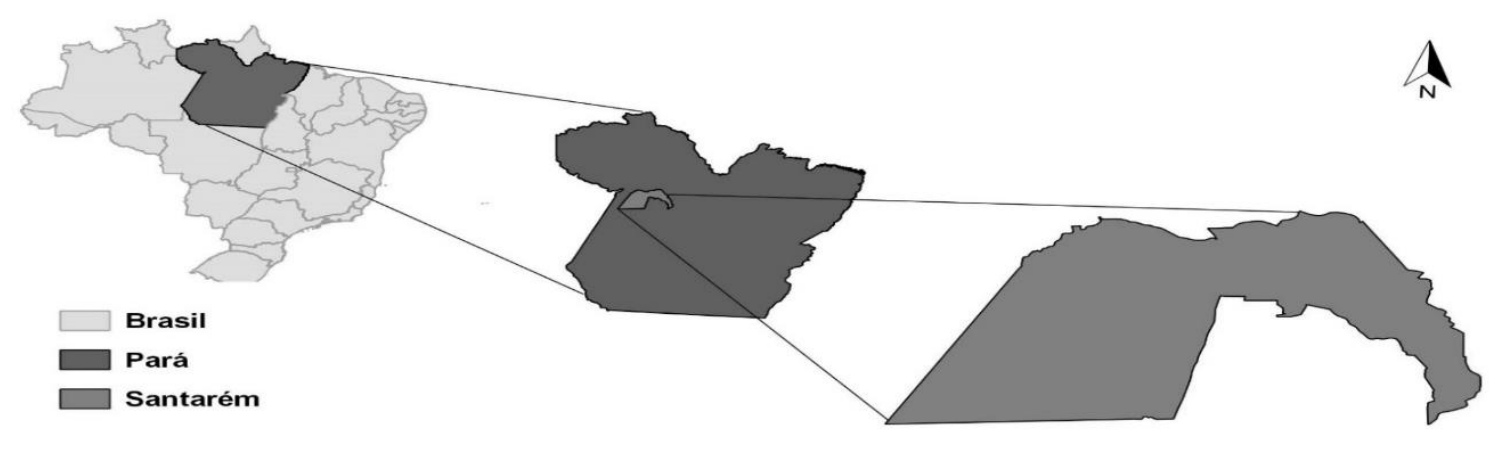

Fonte: Próprios autores.

\section{RESULTADOS}

Primeiramente, foi observado que os dados da DIVISA e SINAN divergiram. No período entre 2012 a 2017 foram notificados $\left(n_{1}=2754\right)$ casos pela DIVISA/Santarém-PA e $\left(n_{2}=2658\right)$ casos no SINAN de acidentes por animais peçonhentos no município de Santarém/PA. 0 ano que apresentou o maior número de ocorrências foi o de 2014, apresentando 187,59 casos para cada 100 mil habitantes. 0 ano de 2012 foi o segundo ano com a maior incidência (184,39 casos/100 mil hab.), seguidos dos anos de 2013, com $(178,45$ casos/100mil hab.), e 2015 com $(81,69$ casos/100mil hab.). Os anos de 2016 e 2017 obtiveram as menores notificações, $(99,85$ casos/100mil hab.) e $(96,18$ casos/100mil hab.) respectivamente (Tabela 1). 
$\mathrm{Na}$ distribuição mensal dos acidentes, o mês de janeiro foi o que apresentou mais casos, seguidos dos meses de setembro e outubro. Os meses com menos acidentes foi dezembro, seguido por junho. Os meses de inverno (dezembro a maio) quando somados apresentaram $51,70 \%$ dos casos, e os meses de verão (junho a novembro) somaram 48,3\% dos casos (Tabela 1 ).

Ao analisar a população notificada por sexo e faixa etária, a maior frequência dos casos dos acidentes ocorreu nos pacientes do sexo masculino $(75,10 \%)$, sendo $(24,9 \%)$ do sexo feminino (Figura 2), com maior ocorrência na idade adulta, 20 a 64 anos (65,39\%), seguidos de 5 a 19 anos (25,13\%), e 65 a 79 anos $(5,42 \%)$. Houve menos casos em indivíduos iguais ou maiores de 80 anos $(0,68 \%)$ e $<1$ a 4 anos (3,38\%) (Figura 2).

Tabela 1 - Distribuição dos acidentes por animais peçonhentos em Santarém (PA), segundo o mês de ocorrência e ano de ocorrência, no período de 2012 a 2017.

\begin{tabular}{cccccccc}
\hline Mês de ocorrência & \multicolumn{7}{c}{ Ano } \\
\cline { 2 - 8 } Janeiro & 2012 & 2013 & 2014 & 2015 & 2016 & 2017 & Total \\
Fevereiro & 55 & 53 & 68 & 51 & 24 & 39 & 284 \\
Marco & 40 & 48 & 49 & 43 & 31 & 23 & 234 \\
Abril & 45 & 45 & 47 & 49 & 18 & 33 & 237 \\
Maio & 46 & 38 & 56 & 45 & 20 & 29 & 234 \\
Junho & 41 & 40 & 38 & 43 & 27 & 24 & 213 \\
Julho & 40 & 31 & 41 & 37 & 19 & 12 & 180 \\
Agosto & 40 & 31 & 46 & 43 & 29 & 22 & 211 \\
Setembro & 36 & 40 & 45 & 42 & 25 & 13 & 201 \\
Outubro & 60 & 47 & 48 & 32 & 23 & 34 & 244 \\
Novembro & 51 & 61 & 32 & 52 & 23 & 25 & 244 \\
Dezembro & 39 & 52 & 34 & 37 & 25 & 17 & 204 \\
Total & 35 & 36 & 41 & 10 & 30 & 14 & 172 \\
\hline
\end{tabular}

Fonte: SINAN, 2019. 
Figura 2 - Distribuição dos acidentes avaliados por sexo e faixa etária, no período de 2012 a 2017, em Santarém (PA).

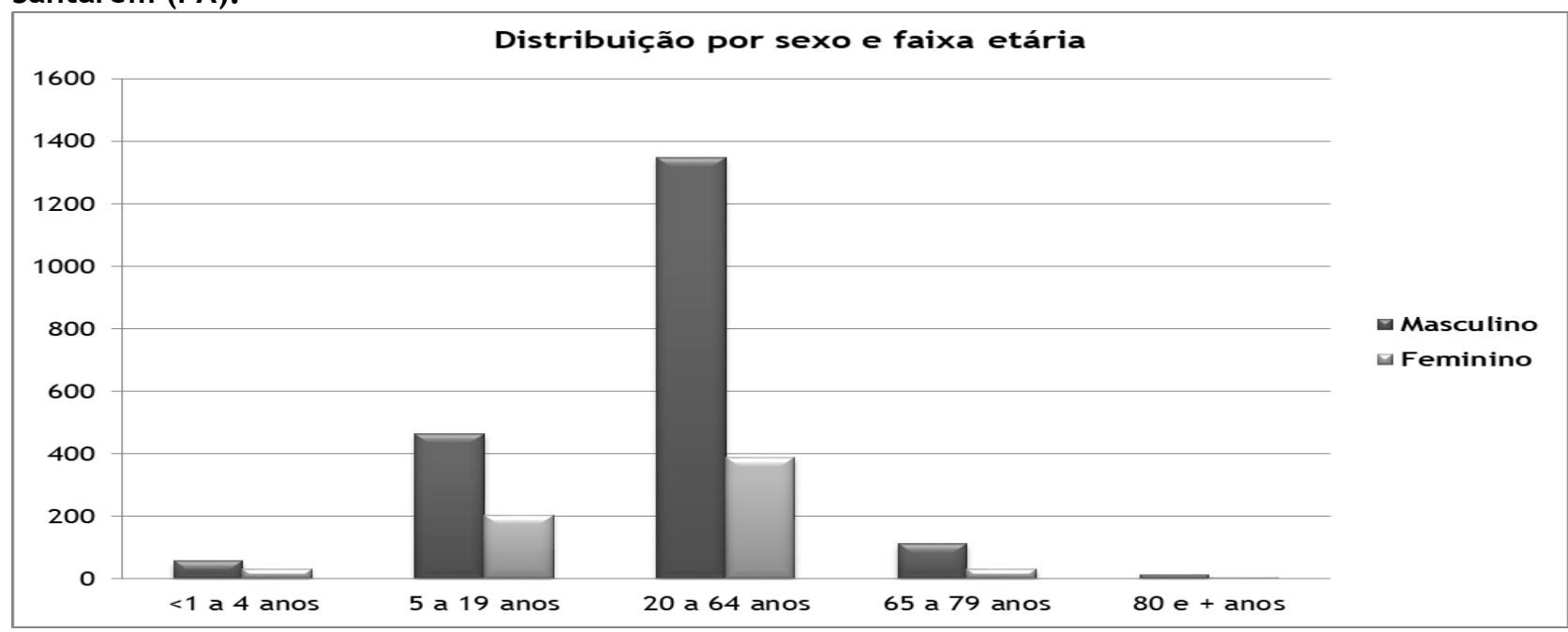

Fonte: SINAN, 2019.

Quanto ao nível educacional das vítimas, (52,71\%) não apresentaram essa informação. Já os casos notificados em que as vítimas possuíam entre $5^{\mathrm{a}}$ e $8^{\mathrm{a}}$ série completa ou incompleta representaram (16,93\%), seguido daquelas que possuíam entre $1^{\mathrm{a}}$ e $4^{\mathrm{a}}$ série completa ou incompleta $(16,70 \%)$. Nos demais níveis educacionais ocorreram menores notificações, sendo $(0,56 \%)$ como analfabetos, $(12,27 \%)$ para o ensino médio completo ou incompleto e $(0,83 \%)$ para o ensino superior completo ou incompleto.

Quanto a localidade de residência das vítimas, a zona rural foi a mais notificada $(68,20 \%)$, seguida pela urbana $(31,00 \%)$. Na região periurbana, área onde as atividades rurais e urbanas se misturam, foram notificados $(0,15 \%)$, e $(0,65 \%)$ foram ignorados ou brancos.
Em relação aos animais causadores dos acidentes, as serpentes apresentaram (42,14\%), e o gênero Bothrops foi o mais representativo $(64,29 \%)$, seguido de Lachesis $(15,71 \%)$, Crotalus (2,14\%), Micrurus $(0,36)$, em $(1,43 \%)$ as serpentes não eram peçonhentas e $(16,07 \%)$ ignorado ou branco. Os escorpiões causaram (35,55\%), aranhas (3,76\%), abelhas $(3,20 \%)$ e lagartas (0,79\%). Os acidentes causados por "outros" animais corresponderam a $(13,77 \%)$, e $(0,79 \%)$ foram ignorados ou brancos.

As regiões anatômicas corpóreas agredidas com maiores registros foram nos membros inferiores, com destaque para o pé com $(49,75 \%)$, seguido por perna com $(13,00 \%)$. Nos membros superiores a mão foi o membro mais acometido com (17,28\%). Os acometimentos na cabeça, braço, 
antebraço e tronco totalizaram $(7,34 \%)$ casos. E $(12,63 \%)$ estavam como ignorados ou brancos.

Os acidentes foram classificados de acordo com o estado clínico dos pacientes como leves (39,35\%), moderados $(37,77 \%)$, graves $(16,82 \%)$ casos e ignorados $(6,05 \%)$ (Figura 3). Nos casos leves analisando o tempo decorrido entre o acidente e o atendimento, $(13,19 \%)$ levaram até uma hora, $(19,98 \%)$ de uma a três horas, $(39,58 \%)$ levaram mais de três horas e $(27,25 \%)$ ignorados. Nos casos moderados
$(10,16 \%)$ levaram até uma hora, $(21,51 \%)$ de uma a três horas, $(56,38 \%)$ mais de três horas e $(11,95 \%)$ ignorados. Nos casos graves $(7,6 \%)$ levaram até uma hora, $(21,92 \%)$ de uma a três horas, $(61,75 \%)$ mais de três horas e $(8,73 \%)$ ignorados (Figura 3).

Os escorpiões e as serpentes obtiveram maiores frequência nos casos graves, $(49,66 \%)$ e $(45,64 \%)$ respectivamente, seguido de "outros" $(3,13 \%)$ e $(1,57 \%)$ foram causados por aranha.

Figura 3 - Distribuição dos acidentes avaliados do tempo percorrido pelo paciente entre a picada e o tratamento e classificação dos casos no período de 2012 a 2017, em Santarém (PA).

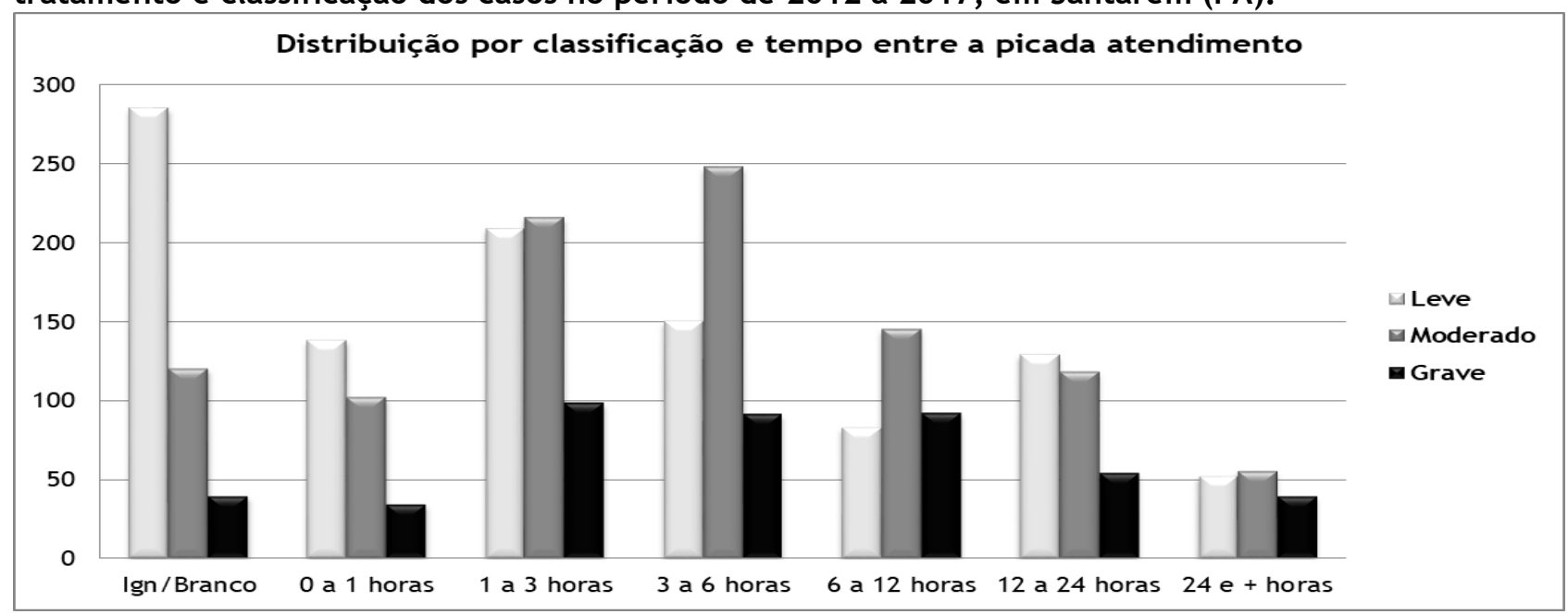

$\mathrm{Na}$ gravidade moderada, as serpentes e os escorpiões foram os animais mais causadores $(51,89 \%)$ e (36,06\%) concomitantemente, (6,97\%) estão como “outros”, $(2,68 \%)$ causados por aranhas, (1,99\%) por abelhas, $(0,30 \%)$ por lagartas e $(0,10 \%)$ ignorado ou branco.
Em relação ao tempo decorrido entre 0 acidente e 0 atendimento, (20,05\%) não registraram essa informação. Dentre os tempos registrados, observou-se que (20,20\%) foram atendidos no intervalo de uma a três horas e $(18,89 \%)$ apresentaram registros de tempo variando de três a 
seis horas. As vítimas que foram atendidas nos intervalos de 6 a 12 h e 12 a 24 horas totalizaram $(12,49 \%)$ e $(11,63 \%)$ casos, respectivamente. Em $(10,80 \%)$ dos pacientes foram atendidos em até uma hora após o incidente e $(5,94 \%)$ levaram 24 horas ou mais pelo atendimento.

Em (86,81\%) dos pacientes apresentaram alguma manifestação clínica local, tais como dor, edema, eritema, calor, parestesia, sudorese ou dor proximal. Enquanto, $(6,14 \%)$ não manifestaram quaisquer sintomas. 0 estudo também reporta que $(7,04 \%)$ foram notificados como ignorados ou deixados brancos na folha de notificação.
As notificações sobre a alteração da coagulação informaram que $(34,82 \%)$ dos pacientes tiveram a coagulação alterada e $(31,77 \%)$ apresentaram normalidade. No entanto, em $(30,47 \%)$ dos pacientes não foi realizado o teste de coagulação, e em (2,94\%) dos registros estavam como ignorados ou brancos.

No que se refere ao uso da soroterapia, o soro antiveneno foi administrado em $(61,76 \%)$ dos casos, enquanto (34,79\%) não foram necessários. Os demais registros $(3,45 \%)$ ignoraram essa informação.

Tabela 2 - Distribuição dos acidentes avaliados do tipo de animal e evolução dos casos no período de 2012 a 2017, em Santarém (PA).

\begin{tabular}{lccccc}
\hline & Ign/Branco & Cura & $\begin{array}{c}\text { Obito pelo agravo } \\
\text { notificado }\end{array}$ & $\begin{array}{c}\text { Óbito por } \\
\text { outra causa }\end{array}$ & Total \\
\hline Ign/Branco & - & 21 & - & - & 21 \\
Serpente & 27 & 1086 & 7 & - & 1120 \\
Aranha & 3 & 97 & - & - & 100 \\
Escorpião & 21 & 922 & 1 & 1 & 945 \\
Lagarta & 2 & 19 & - & - & 21 \\
Abelha & 2 & 83 & - & - & 85 \\
Outros & 9 & 357 & - & - & 366 \\
Total & 64 & 2585 & 8 & 1 & 2658 \\
\hline
\end{tabular}

As maiorias dos pacientes evoluíram para cura $(97,25 \%)$, por outro lado oito pacientes $(0,30 \%)$ vieram a óbito, $(2,41 \%)$ foram notificados como ignorados ou brancos e um $(0,04 \%)$ teve o óbito por outra causa (Tabela 2). Correlacionando as mortes ao tempo percorrido entre $\mathrm{o}$ acidente e o 
atendimento, um óbito levou até 1 hora, três de 1 a 3 horas e cinco levaram mais de 3 horas.

O Hospital Municipal de Santarém (HMS) é considerado um hospital de portas abertas, por ser responsável por uma grande parte da demanda do Oeste do Pará. Atendendo pacientes de baixa e média complexidade, possui 130 leitos, incluindo Unidade de Terapia Intensiva. É referência na região do Baixo Amazonas em atendimentos de vítimas por animais venenosos. Esses pacientes são atendidos por médicos infectologistas e colaboradores, que seguem um fluxo de manejo disponibilizado pelo Ministério da Saúde, que obedecem a alterações específicas de acordo com cada região (Figura 4).

Os pacientes de urgência/emergência acidentados por animais peçonhentos são direcionados ao atendimento médico para avalição e identificação do agressor, sem a necessidade de um cadastro no primeiro momento ou barreira que impeça a rapidez do atendimento. Os pacientes com sintomas leves são mantidos em observação por no mínimo oito horas.

Figura 4 - Fluxograma do manejo clínico para acidentes por animais peçonhentos para o uso de soroterapia específica realizado no Hospital Municipal de Santarém (HMS).

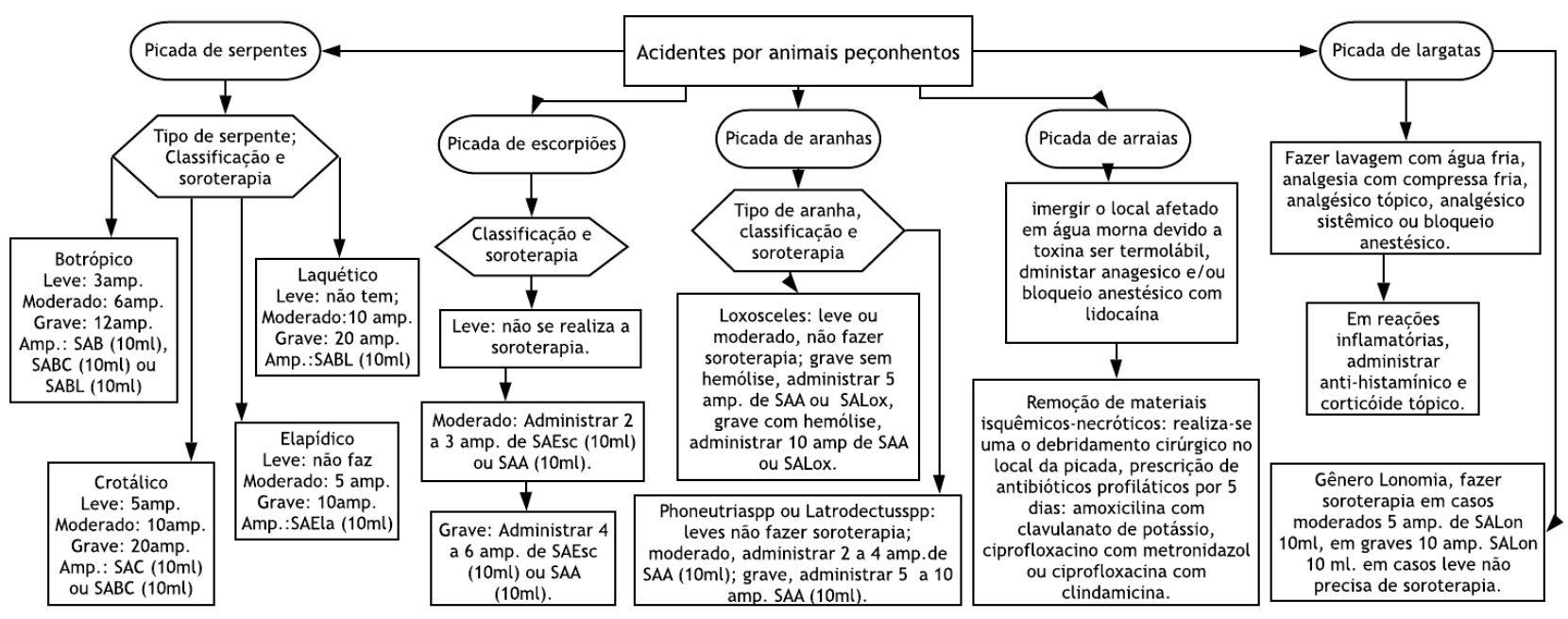

Fonte: HMS, 2019.

Em quadro sugestivo para picada de cobra, algumas condutas são importantes como a elevação membros afetados a $45^{\circ}$ (caso seja nos membros superiores utiliza-se a tipoia); avaliação os sinais vitais e balanço hídrico, higienização do local da picada, sem curativo oclusivo; uso de analgésicos; e coleta de sangue para a realização de exames laboratoriais: hemograma 
(HMG), Ureia (UR), Creatinina (CR), eletrólitos, coagulograma e creatina quinase total (CK).

Para a neutralização do veneno são utilizados os antibotrópicos pentatavalente ( $\mathrm{SAB}$ ) para acidente por Bothrops jararaca; soro antibotrópico pentavalente e antilaquético (SABL) para Lachesis muta e Bothrops jararaca; $(\mathrm{SABC})$ soro antibotrópico pentavalente e anticrotálico soro anticrotálico para Bothrops jararaca e Crotalus durissus; (SAC) para Crotalus durissus; e o soro antielapídico (SAELa) contra Micrurus lemniscatus.

Em casos de reações adversas ao soro antiveneno, deve-se interromper a infusão do soro e utilizar um antihistamínico, se a reação for leve (urticária, hiperemia conjuntival, tosse, edema, dispneia); em uma reação mais grave (hipotensão, síncope, insuficiência respiratória ou circulatória), utiliza-se adrenalina intramuscular (IM) ou intravenosa (IV); após a melhora do paciente, retoma-se ao tratamento com o soro antiofídico. Em alguns casos críticos e/ou graves, recomenda-se fazer na "sala de estabilização" com assistência 24 horas.

Nos acidentes causados por escorpiões, quando os sintomas são apenas locais, como dor local, edema, hiperemia, sensação de "choque" no membro, parestesia e dormência, não se realiza a soroterapia. 0 tratamento é feito apenas com analgésicos. No entanto, o caso é reclassificado se apresentar manifestações sistêmicas. Nos casos moderados, o paciente apresenta sintomas sistêmicos como mioclonias discretas e disartria leve. 0 tratamento consiste na aplicação de duas a três ampolas de antiescorpiônico (SAEsc) ou antiaracnídico (SAA). No entanto, se a vítima apresentar mioclonias intensas ou generalizadas, disartria, ataxia, alteração de pares cranianos, convulsões ou alterações cardiovasculares, edema pulmonar agudo e choque, o caso é considerado como grave, e neste caso, são necessárias de quatro a seis ampolas. Nos casos de mioclonia, também se considera o uso de diazepam (5 a 10mg em adultos e 1 a $2 \mathrm{mg} / \mathrm{kg}$ em crianças) e hidratação intravenosa para manter 0 balanço hídrico.

Em ataques por Loxosceles spp. (aranha-marrom) em gravidade leve e moderado, a administração do soro não é necessária. Contudo, no quadro clínico grave sem hemólise, utiliza-se cinco ampolas de SAA. Quando ocorre hemólise, aplica-se dez ampolas de SAA ou antiloxoscélico trivalente (SALox). Em 
casos picados por Phoneutria spp. (armadeira) ou Latrodectus spp. (viúvanegra), nos casos leves não é realizada a soroterapia. Em quadro moderado, devem ser realizadas aplicações de duas a quatro ampolas de SAA. Em quadro grave, aplica-se cinco a dez ampolas de SAA.

\section{0 tratamento em acidentes} causado por arraias consiste em realizar a assepsia com água potável ou soro fisiológico. Imergir o local afetado em água morna desnatura a toxina por ser uma substância termolábil. Para remoção de materiais isquêmicosnecróticos, realiza-se uma o debridamento cirúrgico no local da picada, com bloqueio anestésico (lidocaína). Após o procedimento de emergência, é indicado a prescrição de antibióticos profiláticos por 5 dias: amoxicilina com clavulanato de potássio, ciprofloxacino com metronidazol ou ciprofloxacina com clindamicina. Na alta do paciente, recomenda-se uma reavaliação.

Em casos de largatas urticantes, o procedimento consiste na lavagem com água fria para remover as espículas e analgesia com compressa fria, analgésico tópico, analgésico sistêmico e bloqueio analgésico. Em casos de reações inflamatórias, deve-se utilizar antihistamínico e corticóide tópico.

Para Lonomia spp. é necessário solicitar exames, a cada 6 a 12 horas, de tempo de coagulação (TC), tempo de protrombina (TP), tempo de tromboplastina parcial ativada (TTPA), relação normatizada internacional (RNI). Também devem ser realizados exames laboratoriais de ureia e creatinina. Se os resultados estiverem normais, o paciente é liberado com tratamento sintomático (analgésicos corticoides tópico e compressa fria). No entanto, se alterados e o paciente apresentar pequenas hemorragias na pele ou mucosa, faz-se uso de cinco ampolas de soro antilonomia. Se o paciente manifestar hemorragia intensas (hematêmese, hematúria) ou Insuficiência Renal Aguda (IRA), fazer dez ampolas de soro antilonômico (SALon).

\section{DISCUSSÃO}

No período em estudo, notou-se uma diminuição acentuada dos casos nos últimos dois anos (2016 e 2017), contrapondo-se ao padrão nacional onde houve um aumento de casos nesse mesmo período ${ }^{18,19}$. Essa diminuição pode estar relacionada ao fato que no 
estado do Pará, a não realização de registros dos acidentes no SINAN é bastante recorrente, não permitindo uma contagem real das notificações, o que se torna um entrave para a adoção de políticas públicas no setor 9 .

Nota-se também uma inconsistência entre os dados cedidos pela DIVISA/Santarém-PA e os retirados do SINAN, demostrando uma possível subnotificação. Essas informações colhidas com omissões rotineiras ou sendo subnotificadas podem gerar uma barreira para se conseguir dados confiáveis a fim de realizar medidas de saúde públicas efetivas?

0 mês de janeiro apresenta 0 maior número de acidentes e este resultado está relacionado ao início da estação chuvosa na região amazônica ${ }^{19}$. Além disso, o mês de junho também apresenta um maior índice de acidentes ofídicos, sendo este período o início da estação com menos chuva na região. As chuvas e o calor fazem que os animais saiam à procura de abrigo, alimento e um ambiente seguro para reprodução ${ }^{8}$.

Os fatores climáticos aliados ao trabalho no campo contribuem para esses incidentes com animais peçonhentos ${ }^{20}$. As principais vítimas são adultas do sexo masculino e faixa etária 20 a 59 anos, devido as suas atividades laborais, agrícolas, pastoris, pesca, lazer e fora do ambiente doméstico ${ }^{21,22}$. A maior ocorrência das notificações se concentra na zona rural ${ }^{23}$. Atividades rotineiras no campo, tais como, extrativismo, pesca, caça e o roçado predispõe a exposição a acidentes por animais peçonhentos ${ }^{15}$.

As serpentes são os principais animais causadores dos acidentes da região norte, seguido dos escorpiões ${ }^{24,14}$. Opondo-se ao perfil epidemiológico de Minas Gerais, por exemplo, que identificou os escorpiões como os principais responsáveis pela maioria dos acidentes, seguido por ofídicos ${ }^{5}$.

Os casos graves foram causados por serpentes, e nos casos com gravidade moderada, eram acidentes ofídicos seguido por escorpiões ${ }^{25,23}$. Em um estudo feito na região do Baixo Amazonas, estado do Pará, observou-se que as espécies de serpentes mais causadoras da região pertencem ao gênero Bothrops, seguido por Lachesis, e também relatam casos dos gêneros, Crotaluse e Micrurus em menores proporções $^{19}$.

Ao analisar a região anatômica de maior ocorrência, os membros inferiores foram os mais atingidos ${ }^{7,12}$. Esses acidentes poderiam ser evitados mediante ao uso de equipamentos de 
proteção individual (EPI) ao realizar atividades rurais, limpeza de jardins, quintais, terrenos baldios e adentrar em matas, roçados e $\operatorname{rios}^{15}$. Ademais, as gravidades dos acidentes podem estar relacionadas a proximidade da picada aos órgãos vitais, além da rápida procura por atendimento e ao tipo de animal causador ${ }^{1}$.

Observa-se que em relação ao nível educacional das vítimas, as notificações são maiores em indivíduos com apenas o ensino fundamental ${ }^{18}$. Apesar dessa variável não ser determinante para as ocorrências, o desconhecimento sobre as medidas preventivas pode predispor uma maior incidência de acidentes ${ }^{25}$.

De acordo com o registro do tempo decorrido entre o acidente e o atendimento, poucos pacientes $(10,80 \%)$ foram atendidos em até uma hora, que corresponde um número baixo em relação aos resultados encontrados em Minas Gerais ${ }^{3,22}$, onde uma boa parte dos pacientes foram atendidos na primeira hora $(48,98 \%) \quad$ e $\quad(43,8 \%)$ respectivamente. Esta procura imediata, todavia, não é regra para todos os casos, principalmente para aqueles que ocorrem em locais distantes do atendimento de saúde ${ }^{12}$.
A distância percorrida dos moradores rurais (rios, floresta e planalto) residentes do município de Santarém ou da região do baixo amazonas para o atendimento na cidade que é polo no atendimento desses agravos, é um dos principais problemas que dificultam as vítimas ao tratamento imediato, pois podem levar horas ou até mesmo dias para chegarem ao HMS, o único que faz o tratamento com soroterapia específica ${ }^{26}$. Quanto menor for o tempo percorrido entre o acidente e o atendimento, melhor será a evolução do paciente ${ }^{3}$. Uma vez identificado o animal causador, se inicia o tratamento imediato, diminuindo o tempo de internação, os riscos de complicações e desenvolvimento de infecções hospitalares.

Nos acidentes ofídicos, a determinação do tempo de coagulação é uma importante medida para auxiliar na confirmação do diagnóstico e avaliação da eficácia da soroterapia ${ }^{10,11,15}$. Segundo os dados colhidos na DIVISA, a maioria dos casos realizou teste de coagulação para o diagnóstico e monitoramento dos pacientes.

O protocolo utilizado na urgência/emergência do Hospital Municipal de Santarém/PA para acidentes provocados por animais 
peçonhentos segue o mesmo preconizado pelo Ministério da Saúde $(M S)^{10,11}$. Os animais agressores são identificados pelas características relatadas pela vítima ou acompanhante e, principalmente, pelo quadro clínico dos pacientes ${ }^{15}$. Segundo esse manejo, os pacientes com manifestação no local da agressão são classificados como leves e não precisam de soroterapia (com exceção quando são ocasionados por serpentes dos gêneros Botrópico, Crotálico, Elapídico e Laquético, que mesmo nos casos leves é preciso a administração da soroterapia) ${ }^{19}$.

o conhecimento clínico dos sintomas é fundamental na classificação dos casos. Os acidentes leves apresentam sintomas transitórios que se resolvem espontaneamente. Enquanto que os acidentes moderados, são mais pronunciados e prolongados. Os acidentes graves apresentam sintomas graves, podendo ocasionar a morte do paciente? ${ }^{2}$.

Os antivenenos são os únicos tratamentos capazes de neutralizar as substâncias tóxicas inoculadas por animais peçonhentos, sendo este o único tipo de tratamento específico indicado pelo Ministério da Saúde ${ }^{16}$. Entretanto, a região em estudo tem o hábito cultural de realizar o tratamento com plantas e/ou outros tipos de remédios medicinais, com o intuído de combater o veneno, conhecidos como "contraveneno", deixando muitas vezes de irem ou tardarem a ida ao Hospital, podendo gerar complicações tardias ${ }^{26}$.

\section{CONCLUSÃO}

O perfil epidemiológico descrito nesse estudo possibilitou uma ampla análise da real situação dos acidentes causados animais peçonhentos no município de Santarém (PA). Foi identificada uma regressão dos acidentes no período avaliado, com uma maior frequência dos casos em pacientes do sexo masculino, em idade economicamente ativa e com maior incidência no período de chuvas (janeiro a junho). Os principais animais causadores foram as serpentes, seguida por escorpiões e aranhas.

Contudo, foram identificadas possíveis falhas na notificação desses acidentes devido à expressividade da categoria "ignorado/branco". Além disso, constatou-se uma inconsistência entre as duas fontes de dados (SINAN e DIVISA), dificultando na obtenção de um resultado mais próximo da realidade. Essas notificações quando bem realizadas produzem indicadores 
epidemiológicos promissores para os gestores em saúde, garantido um melhor direcionamento dos recursos terapêuticos, de acordo com a realidade da região.

\section{REFERÊNCIAS}

1. Beraldo HS, Anchieta DW, Kupka FS, Maraschin MS, Alves $\mathrm{DCl}$. Acidentes com animais peçonhentos notificados em um hospital escola. Rev Varia Scientia. 2017; 3(2):194200.

2. Silva JH, Giansante S, Silva RCR, Silva G, Silva LB, Pinheiro CBL. Perfil epidemiológico dos acidentes com animais peçonhentos em Tangará Da Serra-MT, Brasil (2007-2016). J Health NPEPS. 2017; 2(1):5-15.

3. Silveira JL, Machado C. Epidemiologia dos acidentes por animais peçonhentos nos municípios do Sul de Minas Gerais. J Health NPEPS. 2017; 2(1):88-101.

4. WHO. Rabies and Envenomings: A Neglected Public Health Issue: Report of a Consultative Meeting, World Health Organization, Geneva, 10 January 2007: World Health Organization; 2007.

5. Silva PLN, Costa AA, Damasceno RF,
Oliveira Neta AI, Ferreira IR, Fonseca ADG. Perfil epidemiológico dos acidentes por animais peçonhentos notificados no Estado de Minas Gerais durante o período de 20102015. Rev SUSTINERE. 2017; $5(2): 199-217$.

6. Ferreira LC, Rocha YCS. Incidência de acidentes por escorpiões no município de Januária, Minas Gerais, Brasil. J Health NPEPS. 2019; 4(1):228-241.

7. Rita TS, Sisenando HA, Machado C. Análise epidemiológica dos acidentes ofídicos no município de Teresópolis - RJ no período de 2007 A 2010. Rev Ciênc Plural. 2016; 2(2):28-41.

8. Cheung R, Machado C. Acidentes por animais peçonhentos na região dos lagos, Rio de Janeiro, Brasil. J Health NPEPS. 2017; 2(1):73-87.

9. Silva JAC, Silva BS, Alves LFR, Dantas MP, Neves VBFF, Carvalho PAF. Incidência de acidentes com animais peçonhentos no estado do Pará. Braz J Health Rev. 2019; 2(2):3313-3317.

10. Ministério da Saúde (BR). Secretaria de Vigilância em Saúde. Guia de Vigilância em Saúde. Brasília: Ministério da Saúde; 2019.

11. Ministério da Educação (BR). Serviço de Doenças Infecciosas e 
Parasitárias. Diretrizes Diagnósticas de Acidentes com Animais Peçonhentos. Rio de Janeiro: Universidade Federal do Rio de Janeiro; 2016.

12. Barbosa IR. Aspectos clínicos e epidemiológicos dos acidentes provocados por animais peçonhentos no estado do Rio Grande do Norte. Rev Ciência Plural. 2015; 1(3):2-13.

13. Ministério da Educação (BR). Instituto Federal do Norte de Minas Gerais. Epidemiologia Aplicada à Saúde Pública. Montes Claros (MG): Ministério da Educação; 2015.

14. Alencar ES, Araújo MHS, Carvalho AV. Acidentes por animais peçonhentos no município de Guaraí (TO) no período de 2015-2017. Medicus. 2019; 1(1):10-21.

15. Ministério da Saúde (BR). Fundação Nacional da Saúde. Manual de Diagnóstico e Tratamento de Acidentes por Animais Peçonhentos. Brasília: Ministério da Saúde; 2001.

16. Souza CMV, Machado C. Animais peçonhentos de importância médica no município do Rio de Janeiro. J Health NPEPS. 2017; 2(1):16-39.

17. Medeiros WRP. Registros de ataques por animais peçonhentos no Hospital Regional de Patos [trabalho de conclusão de curso]. Patos: Universidade Federal de Campinas, Departamento de Ciências Biológicas; 2014.

18. Ministério da Saúde (BR), Secretaria de Vigilância em Saúde. Acidentes de trabalho por animais peçonhentos entre trabalhadores do campo, floresta e águas. Brasília: Ministério da Saúde; 2019.

19. Silva MJC, Soares FT, Trindade GP, Diniz HS, Medeiros JMR, Lopes JGM, et al. Perfil epidemiológico dos acidentes ofídicos da mesorregião do baixo Amazonas do estado do Pará, Brasil. Braz J Heath Rev. 2019; 2(4):1968-1979.

20. Oliveira HFA, Costa CF, Sassi R. Relatos de acidentes por animais peçonhentos e medicina popular em agricultores de Cuité, região do Curimataú, Paraíba, Brasil. Rev Bras Epidemiol. 2013; 16(3):633-643.

21. Machado C. Um panorama dos acidentes por animais peçonhentos no Brasil. J Health NPEPS. 2016; 1(1):1-3.

22. Ladeira CGP, Machado C. Epidemiologia dos acidentes com animais peçonhentos na região de Ponte Nova, Minas Gerais, Brasil. J Health NPEPS. 2017; 2(Supl.1):40-57. 
23. Assis SNS, Rodrigues JJP, Lima RA. Levantamento de acidentes com animais peçonhentos registrados em Tabatinga-AM, Brasil. Rev Gest Sust Ambient. 2019; 8(1):582-599.

24. Lopes AB, Oliveira AA, Dias FCF, Santana VMX, Oliveira VS, Liberato AA, et al. Perfil epidemiológico dos acidentes por animais peçonhentos na região norte entre os anos entre 2012 e 2015. Rev Patologia Tocantins. 2017; 4(2):36-40.

25. Santana VTP, Suchara EA. Epidemiologia dos acidentes com animais peçonhentos registrados em Nova Xavantina - MT. Rev Epidemiol Control Infect. 2015; 5(3):141-146.
26. Silva EMR. Acidentes escorpiônico no município de Santarém-PA: característica epidemiológica e trajeto percorrido pelos pacientes até o serviço de saúde [dissertação]. São Paulo: Universidade de São Paulo, Escola de Enfermagem; 2017.

Financiamento: Os autores declaram que não houve financiamento.

Conflito de interesses: Os autores declaram não haver conflito de interesses.

\section{Participação dos autores:}

- Concepção: Lopes LD, Lisbôa JDB, Silva FG.

- Desenvolvimento: Lopes LD, Lisbôa JDB, Silva FG.

- Redação e revisão: Lopes LD, Lisbôa JDB, Silva FG.

Como citar este artigo: Lopes LD, Lisbôa JDB, Silva FG. Perfil clínico e epidemiológico de vítimas de acidentes por animais peçonhentos em Santarém - PA. J Health NPEPS. 2020; 5(2):161-178.

Submissão: $12 / 07 / 2020$

Aceito: $02 / 10 / 2020$

Publicado: 04/12/2020 